\title{
Factores asociados a la elaboración de tesis en la Universidad Nacional de Asunción, Paraguay
}

Factors associated to the elaboration of thesis at the Universidad Nacional de Asunción, Paraguay

\author{
Volumen 20, Número 2 \\ Mayo - Agosto \\ pp. 1-24
}

\section{Carmiña Hilda Soto Figueredo}

Soto Figueredo, Carmiña Hilda. (2020). Factores asociados a la elaboración de tesis en la Universidad Nacional de Asunción, Paraguay. Revista Actualidades Investigativas en Educación, 20(2), 1-24. Doi. 10.15517/aie.v20i2.41676 


\title{
Factores asociados a la elaboración de tesis en la Universidad Nacional de Asunción, Paraguay \\ Factors associated to the elaboration of thesis at the Universidad Nacional de Asunción, Paraguay
}

\section{Carmiña Hilda Soto Figueredo ${ }^{1}$}

\begin{abstract}
Resumen: Se muestran algunos resultados de la investigación contenida en la tesis doctoral de la autora, cuyo objetivo es indagar cuáles son los factores asociados a la elaboración de tesis de maestrías y doctorados en la Universidad Nacional de Asunción, en un periodo de análisis de 10 años (2004-2014). Se llevó a cabo una investigación mixta, de diseño correlacional retrospectivo y transversal, con una muestra no probabilística de 189 egresados y egresadas. Los resultados dan cuenta de una asociación entre trabajo a tiempo total/parcial $X^{2}(1$, $N=189)=5,934, p=0,018$, becas $o$ ayudas monetarias $X^{2}(1, N=189)=12,45 p=0,0001$, docencia e investigación $X^{2}$ $(4, N=189)=20,44, p=0,0001$, frecuencia de encuentro con el tutor o la tutora de tesis $X^{2}(3, N=189)=84,6, p=0,001$ y realización de tesis; por otro lado, no se encontró asociación entre el sexo, edad al matricularse, existencia de comités o asesorías de tesis, realización de cursos o talleres de tesis, publicaciones y elaboración de tesis. El principal factor (22\%) que impide la realización de tesis es la cuestión metodológica (el o la tesista no sabe cómo hacer la tesis, tiene problemas con la escritura). Las entrevistas evidenciaron la desmotivación en el estudiantado respecto a la falta de acuerdo entre el cuerpo de evaluadores, tutores y mesa examinadora. Se pudo visualizar el carácter complejo y multifactorial que lleva a una situación de no elaboración de la tesis, donde la práctica y experiencia previas en trabajos científicos y en la enseñanaza universitaria, puede facilitar la finalización de la tesis $(p=0,0001)$.
\end{abstract}

Palabras clave: tesis, investigación, posgrado, enseñanza superior

\begin{abstract}
Some results of the research contained in the author's doctoral thesis are shown around the factors associated with the preparation of master's and doctoral theses, where the selection of the content made for this article, was the aim was to analyze the factors associated with the elaboration of master and doctoral's thesis from Universidad Nacional de Asunción, taking data from the 2004-2014. A quali-quantitative study was carry out, with analytical, retrospective and cross-sectional design, with a non-probabilistic sample of 189 research subjects. Significant association between part/full time work $X^{2}(1, N=189)=5,934, p=0,018$, scholarship $X^{2}(1, N=189)=12,45$ $p=0,0001$, teaching and research activity $X^{2}(4, N=189)=20,44, p=0,0001$, meeting with the advisor $X^{2}$ (3, $N=189)=84,6, p=0,001$ and make the thesis were found; and not significant association between sex, age to begin de postgraduate program, thesis committee o advisor, making curse or workshop about thesis, publication and to make de thesis was found. In both, as the main factor that prevents the realization of the thesis, was methodological question (22\%), it does not how to do it, has problems with writing. The interviews showed the demotivation of students regarding the lack of agreement between evaluators, tutors and jury. It was possible to visualize the complex and multifactorial character that leads to a non-thesis situation.
\end{abstract}

Key Words: thesis, research, postgraduate, higher education

\footnotetext{
${ }^{1}$ Docente de la Universidad Nacional de Asunción, Paraguay, Dirección General de Postgrado y Relaciones Internacionales. Doctora en Educación, con Énfasis en la Gestión de la Educación Superior por la Universidad Nacional de Asunción.
}

Dirección electrónica: carmina.soto@gmail.com ORCID_https://orcid.org/0000$\underline{0003-0522-9915}$

Artículo recibido: 16 de octubre, 2019

Enviado a corrección: 15 de febrero, 2020

Aprobado: 20 de abril, 2020 


\section{Introducción}

En América Latina, la cultura de la investigación, entendida la última como producción de nuevos conocimientos al interior de las universidades, es aún baja comparada con Estados Unidos, Alemania o Japón por ejemplo, donde se conciben las universidades como "centros de producción intelectual y donde todo personal docente universitario cumple un papel fundamental en este proceso" (Narváez y Burgos, 2011, p.117).

Los programas de postgrados, especialmente los doctorados, deben servir para generar investigación científica propiamente dicha y las maestrías para formar profesionales en investigación científica al interior de las universidades (Peñaloza, 2003). Como un requisito para la obtención del grado académico de máster o doctor, se encuentra la elaboración de la tesis, y a pesar de la trascendencia del acto académico, la eficiencia terminal (estudiantes que logran el grado académico una vez elaborada y defendida la Tesis) es baja-aunque el alumnado haya terminado de cursar el Plan de Estudios-pues no sobrepasa especialmente en Latinoamérica, el 10\% (Martínez et al., 2003; Peñaloza, 2003; Soto, Velázquez y Samudio, 2014).

El éxito académico, traducido en la obtención del título de postgrado, además de otorgar cierto reconocimiento entre colegas y conferir mayor prestigio y notoriedad a la persona profesional y a la casa de estudio que le dio la educación, constituye en la Sociedad del Conocimiento un requisito indispensable para pretender mejores accesos laborales, donde toda persona con menor oportunidad de acceso a mayores niveles educativos quedará relegada, de acuerdo con la Teoría del capital humano, que dice que la persona con estudios universitarios obtendrá mayores beneficios a futuro (Rodríguez, 2014).

En la Universidad Nacional de Asunción, la eficiencia terminal en los programas de postgrado también es baja. Un estudio piloto realizado por Soto et al., (2014) encontró que sólo un 5,3\% del estudiantado matriculado en programas de postgrado entre los años 2005 y 2008 en seis unidades académicas, se graduaron, es decir culminaron la tesis de manera exitosa.

Esta situación generó la pregunta ¿cuáles son los principales factores por los cuales las personas, aun habiendo terminado de cursar la malla curricular, no elaboran la tesis de maestrías y doctorados en la Universidad Nacional de Asunción? Encontrar la respuesta sobre los principales factores que impiden o retrasan lograr la eficiencia terminal del estudiantado de postgrado constituye el objetivo de esta investigación. 
Disponer de datos empíricos que revelen la situación de los estudiantes al momento de elaborar la tesis brindará elementos de gestión que ayuden a revertir la baja eficiencia académica del sistema de estudios de postgrado, con lo que se evitarán frustraciones, "síndromes" y hasta deserciones en los programas. Además, si una persona que cursa un postgrado culmina exitosamente sus estudios se genera una dinámica socioeducativa con la disponibilidad de nuevos conocimientos, lo que da origen a nuevas investigaciones.

\section{Marco Teórico}

La demora excesiva o la no elaboración del trabajo de tesis ha originado lo que se denomina: El Síndrome Todo Menos Tesis (TMT).El término TMT fue acuñado por Elizabeth Valarino en el año 1987, quien tomó como información base la traducción All But Dissertation donde Cone y Foster, en la década de los años noventa, hacían referencia a estudiantes que no concluían la tesis doctoral (Valarino, 2000).

El Síndrome TMT impide lograr el éxito académico, entendido como la obtención del grado académico (en este caso de máster o doctor) de los cursos de postgrado (Martínez et al., 2003; Valarino, 2000). A decir de Valarino (2000), "es frecuente encontrar en tesistas o en personas que realizan investigaciones algunas sintomatologías clínicas como la depresión, enfermedades psicosomáticas, irritabilidad y estados nerviosos y, en casos graves, ciertos rasgos histéricos o paranoides" (p.155). De allí, la denominación de "síndrome" (patología general - síntomas y signos que concurren en una enfermedad), lo que hace que la atención a las personas que trabajan en la elaboración de la tesis requiera de un trabajo multidisciplinario y sistemático (Valarino, 2000, p.7)

Los factores que llevan a una situación de TMT pueden ser amplios, variados y complejos. Se estudiaron las implicancias de los factores académicos, personales, sociales, cognitivos, emocionales, institucionales, y la interrelación entre factores, en las que los factores académicos y personales son los más estudiados (Valarino, 2000). Así Rietveldt de Arteaga y Vera (2012), en un estudio realizado en la Universidad del Zulia-Venezuela, encontraron que solo el 35\% del estudiantado que ingresa a un postgrado logra cursar y aprobar todas las asignaturas de este. Valarino (2000) encontró, entre los factores o causas principales que reducen el Síndrome TMT: la dedicación a tiempo completo, el financiamiento externo, la preparación y la experiencia de todo el personal docente de investigación. También, menciona que el buen rendimiento durante el desarrollo del 
programa de postgrado parece favorecer a un reducido número de estudiantes al momento de elaborar la tesis.

Asimismo, un estudio realizado en el Instituto de Tecnología de Georgia por Jacks et al., (citado en Valarino, 2000, p.135) encontró como causa principal de la no elaboración o finalización de la tesis entre las personas candidatas a doctor, a más del problema financiero, una "pobre relación con el tutor o la tutora". Peñaloza (2003, p.147), en tanto, sostiene que el bajo nivel de las personas graduadas en los cursos de postgrados se debe básicamente al diseño de la malla curricular donde los cursos de postgrado siguen una modalidad de "cursos panorámicos", propio de los cursos de grado, por lo cual el estudiantado no lleva a cabo investigaciones, no sabe investigar, traduciéndose en postergaciones en la elaboración de la tesis.

Por otro lado, y según Rojas (2008), la formación temprana de jóvenes en investigación, a fin de adquirir la habilidad en la producción científica, constituye un problema pedagógico debido, entre otros aspectos, a la baja capacidad de las propias instituciones de enseñanza superior en generar la producción de conocimientos, investigaciones y la conformación y visibilidad de grupos de investigación lo cual tiene implicancias al momento de elaborar un trabajo científico, como la tesis.

Ampliando la mirada sobre los factores, Klubitschko (citado en Valarino, 2000) refiere que el atraso en las investigaciones en América Latina principalmente se debe a la estructura económico-social, que no demanda los servicios derivados de la investigación. Valarino, (2000) así como Peñaloza (2003) coinciden en que la mayoría del estudiantado que se inscribe en cursos de maestrías no persigue ser investigador. Así Klubitschko (citado en Valarino, 2000) encontró que un 35\% de personas encuestadas manifestó como principal motivación para ingresar a los programas de postgrado, la investigación; y, más bien desean mejorar sus destrezas profesionales, produciéndose una desvirtuación de lo que implican los cursos de maestrías, donde se debe preparar para la investigación; y los doctorados, donde el objetivo debe ser la producción científica (Peñaloza, 2003).

Las condiciones precarias tanto de la infraestructura así como las condiciones bajo las cuales se produce la vinculación con el cuerpo docente de postgrado, de manera transitoria sin vinculación directa a los objetivos institucionales, podrían constituir también factores que restan motivación al estudiantado de los postgrado en la elaboración de la tesis. Sumado a lo anterior, la falta de un mecanismo de seguimiento eficiente al alumnado así como la carencia en cuanto a habilidades básicas para procesar la información, podrían ser consideradas 
como causas que influyen en el problema TMT (Sánchez, citado en Rietveldt de Arteaga y Vera, 2012).

En los países desarrollados, aunque el porcentaje de eficiencia terminal es mayor que en América Latina, tampoco el problema Todo Menos Tesis se halla ausente. Un trabajo realizado por Kris Latona y Mairead Browne de la University of Tecnologyde Sydney, menciona como factores que influyen en la elaboración de la tesis de postgrado, "las diferencias entre las disciplinas y las características de los requisitos de admisión, el tipo de supervisión realizado y las características generales de las cohortes y de las individuales relacionadas con estudiantes, sus percepciones y sus modos de comportarse" (Hirschorn, 2012, p.20).

Otro estudio realizado en el Reino Unido, tuvo por objetivo indagar experiencias y estrategias que faciliten el proceso de aprendizaje de redacción de tesis, de modo de superar las estadísticas que indican que el $75 \%$ de los estudiantes de doctorado completan la tesis después de los cuatro años esperados; y en este sentido se discuten aspectos concernientes con los supervisores, organización personal y estrategias de aprendizaje. Los resultados indican que el estudiantado percibe como beneficioso para el proceso de tesis la retroalimentación de apoyo individualizada y la importancia de la capacidad de recuperación de los estudiantes (Odena y Burgess, 2017).

En tanto, Locke y Bolyle (2016) también mencionan el problema TMT diciendo que más de la mitad del alumnado de programas de postgrado abandonan el proceso sin elaborar la tesis y en este sentido realizaron un estudio de corte cualitativo donde recabaron la percepción del alumnado de un programa de doctorado en liderazgo educativo encontrando que las causas de este abandono se relacionaban con la disponibilidad de tiempo, la dificultad con la escritura y con el asesoramiento.

Como se observa, el interés por entender el fenómeno Todo Menos Tesis es creciente y se vuelve cada vez más estudiado. Carlino (2005), en su investigación denominada La experiencia de escribir una tesis: contextos que la vuelven más difícil, cuyos hallazgos fueron presentados en el II Congreso Internacional de Cátedra UNESCO Lectura y Escritura, menciona que existen algunos factores que facilitan la escritura de la tesis y otros en cambio lo dificultan. La autora agrupa estos factores en cinco considerados relevantes, aunque no son independientes entre sí, que acorde se hallen de manera positiva o negativa juegan un rol decisivo en la elaboración de la tesis. 
Los factores mencionados son: 1) la pertenencia a una u otra tradición disciplinar (aquellos que pertenecen a las ciencias no exactas o no naturales tropiezan con mayores dificultades); 2) la inclusión o no en un equipo de investigación. La falta de "provisión de sostén" dificulta adentrarse en la comunidad de práctica (la tesis) que de por sí ya resulta desconocida y desafiante para la mayoría del estudiantado; 3) la dedicación a tiempo completo o parcial; 4) el tener o no práctica previa en investigación y 5) el tener o no un director, directora especialmente dedicado o dedicada (Carlino, 2005, p.3).

También menciona como factores que ayudan a la elaboración de la tesis los espacios donde se puedan compartir los sentimientos comunes a la elaboración de la tesis y donde se puedan sobrellevar los sentimientos de aislamiento y desánimos que en ocasiones enfrenta quien realiza la tesis. A todo lo anterior se suma el auge experimentado por los programas de postgrado, principalmente a partir de los noventa, tanto en lo que respecta a la cantidad de las instituciones oferentes, como la cantidad de las personas matriculadas, así como de la oferta misma de los programas lo cual significó para las instituciones de educación superior un desafío, tanto en lo concerniente a la capacitación del cuerpo docente, como las adecuaciones edilicias, cambios en los programas y mallas educativas así como en los aspectos tecnológicos; requerimientos que aún se llevan implementados en las universidades y los cuales influyen al momento de lograr una mayor eficiencia terminal de estudiantes de postgrado y reducir la categoría TMT.

El éxito académico, traducido en la obtención del título de postgrado, no solo otorga cierto reconocimiento entre colegas al conferir mayor prestigio y notoriedad profesional, sino también a la casa de estudio que le dio la educación, lo cual constituye en la Sociedad del Conocimiento, un requisito indispensable para propender a mejores accesos laborales, donde quedarán relegados quienes tienen menor oportunidad a mayores niveles educativos, que conteste con la Teoría del capital humano, la persona con estudios universitarios obtendrá mayores beneficios a futuro (Rodríguez, 2014).

\section{Materiales y Métodos}

\subsection{Enfoque}

La investigación es de tipo mixto, con un diseño observacional y trasversal cuyo alcance fue descriptivo con componente analítico. La utilización de los diseños de método mixto se constituye en una buena alternativa para abordar temáticas de investigación en el campo educativo, donde se pretende lograr la comprensión de fenómenos más que objetos y 
la investigación mixta permite incorporar narraciones o verbalizaciones de los actores, que ofrecen mayor sentido a los datos numéricos (Pereira, 2011).

\subsection{Unidad de análisis}

La población objetivo estuvo integrada por personas egresadas que terminaron de cursar el Plan Curricular del Programa de Postgrado, pero no han finalizado el proceso de elaboración, presentación, defensa y aprobación del trabajo de tesis y personas graduadas (quienes obtuvieron el grado académico-máster o doctor, doctora) de programas de maestrías y doctorados cuyos criterios de inclusión fueron haber cursado estos programas en la Universidad Nacional de Asunción entre los años 2004 y 2014, registrándose 1609 estudiantes. En tanto se consideró como población accesible aquellas personas egresadas y graduadas a cuyos datos de contacto (dirección de correo electrónico) se pudo acceder, atendiendo la modalidad electrónica de administración de las encuestas, que representó $86 \%$ respecto a la población total, con una nueva merma de aproximadamente un $10 \%$,debido a direcciones de correos invalidadas.

La remisión del cuestionario se realizó al total de la población accesible, 1249 personas. La cantidad de persona encuestada que respondió el cuestionario quedó constituida por 189, provenientes de 9 unidades académicas (de las doce existentes en el año de realización del trabajo), siendo esta la muestra de la investigación. Coincidente con Díaz de Rada (2012), quien sostiene que la desventaja de administrar encuestas vía electrónica radica en el bajo índice de respuestas, y en este caso dicha cifra representó un $14 \%$. Al no tener todas las personas la misma oportunidad de formar parte de la muestra, debido a que no se accedió a la dirección de correo electrónico del total de la población, la muestra fue no probabilística. Para las entrevistas se seleccionó a 6 actores, 2 tutores o tutoras, 2 graduados o graduadas y 2 egresados o egresadas, y fue por conveniencia.

\subsection{Técnicas de recolección}

La técnica de recolección de información fue la encuesta y se utilizó como instrumento de recolección de datos un cuestionario estructurado conformado por 15 indicadores (tabla 1) destinados a la evaluación de los factores asociados a las dimensiones personales, académicas e institucionales, de los cuales 5 ítems fueron de caracterización sociodemográficas (género, edad, procedencia, edad de inicio, estado civil). La forma de administración del cuestionario fue vía electrónica mediante la plataforma Google Drive. La 
encuesta se realizó entre noviembre de 2016 y julio de 2017.Para el componente cualitativo, se utilizó la entrevista semiestructurada a dos tutor o tutora; dos personas graduadas (con tesis) y 2 personas egresadas (sin tesis).

Validez y fiabilidad: Las variables mencionadas en la Tabla 1 constituyen algunas que formaron parte de un cuestionario más extenso que pasó por el proceso de validación mediante la revisión bibliográfica, juicio de expertos y una prueba piloto aplicada a 32 personas egresadas y graduadas de maestrías ofrecidas por la Dirección General de Postgrado y Relaciones Internacionales del Rectorado (UNA), por el Centro Multidisciplinario de Investigaciones Tecnológicas, también del Rectorado, y la Facultad de Ciencias Agrarias. La encuesta piloto se aplicó durante los meses de noviembre de 2016 y febrero de 2017.

La validación de constructo se realizó mediante el análisis factorial que arrojó un valor de $\mathrm{KMO}=0,585>0,5$ lo cual es indicativo de correlación y, por lo tanto, afirma que el análisis factorial es una técnica útil para el estudio. En la Prueba de Bartlett, el valor asociado al estadístico Chi cuadrado se mostró significativo $X^{2}(21, N=27)=51,69, p<0,05$ con lo que se rechaza la $\mathrm{H}_{0}$, existiendo correlaciones entre variables. En tanto, el cálculo de fiabilidad del instrumento para el grupo de variables de la Escala tipo Likert arrojó un alfa corregido de Cronbach de $\alpha=0,739$. 
Tabla 1

Operacionalización de variables del estudio para las personas egresadas de la Universidad Nacional de Asunción-Paraguay del 2004-2014

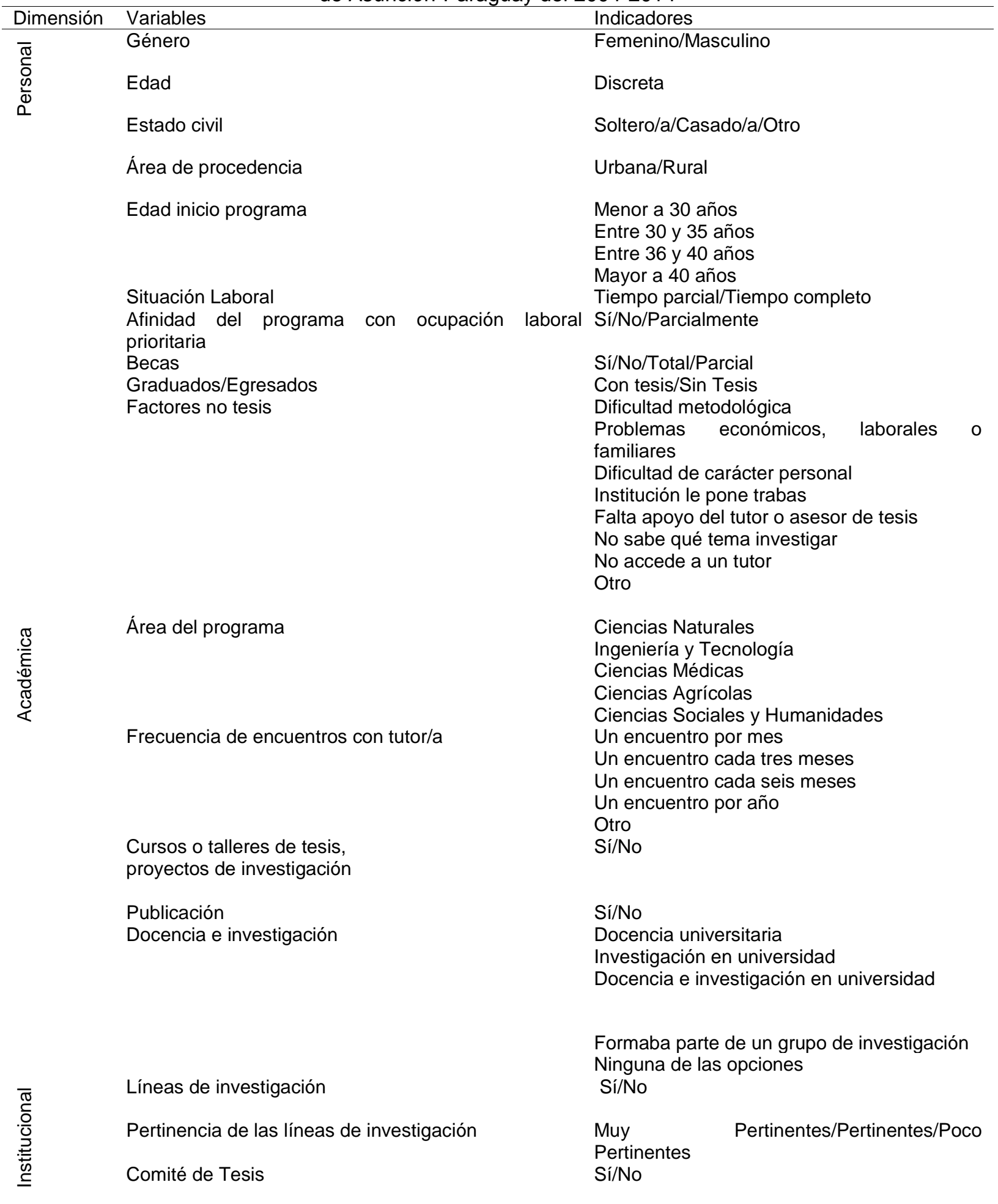

Fuente: Elaboración propia 


\subsection{Procesamiento de análisis}

El procesamiento y análisis de la información para el segmento descriptivo se realiza mediante frecuencias y porcentajes mientras que para el análisis de contraste de hipótesis se utilizó la prueba Chi cuadrado para investigar el grado de independencia de las variables cualitativas, considerándose como significativa una $p$ de $0,05(p<0,05)$. El análisis se realizó en el programa estadístico SPSS versión 15.0. Para el segmento cualitativo, es decir las entrevistas, después del consentimiento verbal de la persona entrevistada, se realizó la grabación y luego el desgrabado y trascripción de la misma, y el análisis llegó a un nivel descriptivo.

\section{Resultados}

Un $52,4 \%$ de las personas encuestadas son de género femenino y $47,1 \%$ masculino $(0,5 \%$ sin respuesta); la procedencia mayoritaria $(52,9 \%)$ fue del área rural. La edad promedio es 40 años ( $\mathrm{ME}=40, \mathrm{DE}=8)$ con un mínimo de 27 años y un máximo de 67 años. El estado civil muestra que el 48,1\% ( $n=91)$ estuvo casado/a al momento de iniciar el postgrado, el $8,5 \%(n=16)$ era soltero/a y el estado civil de un $43,4 \%(n=82)$ fue de otras modalidades (divorciado/a, conviviente, unido/a). Indagando respecto a la universidad donde han cursado la carrera de grado, el 85,2\% respondió que lo hizo en una universidad pública.

En la tabla 2 se visualiza que un $46,0 \%(n=87)$ no realizó la tesis y pasa a pertenecer a la categoría TMT y un 53,9\% lo realizó $(n=102)$. El porcentaje entre varones y mujeres con tesis así como entre mujeres y varones sin tesis son similares, y las diferencias no son significativas $\mathrm{X}^{2}(1, \mathrm{~N}=189)=0,244, \mathrm{p}=0,621$. Puede observarse que los programas de postgrado deben enfrentar el serio problema de la no realización de la tesis, por lo que estas personas pasan a formar parte del denominado ABD-en inglés (All But Dissertation-TMTTodo Menos Tesis) es decir, estudiantes que han finalizado de cursar la malla de la maestría o doctorado y no han hecho la tesis, y caen según Hirschhorn (2012), en la categoría de educación informal. La misma autora menciona que los varones tienen $24 \%$ más chances de caer en la categoría TMT. 
Tabla 1

Número y porcentaje de estudiantes que defendieron tesis, por género, Universidad Nacional de Asunción-Paraguay 2004-2014

\begin{tabular}{cccccc}
\hline Género & \multicolumn{2}{c}{ Con tesis } & \multicolumn{2}{c}{ Sin tesis } & Total \\
& Frecuencia & Porcentaje & Frecuencia & Porcentaje & \\
\hline Femenino & 52 & 51 & 47 & 54 & 99 \\
Masculino & 50 & 49 & 40 & 46 & 90 \\
Total & 102 & 100 & 87 & 100 & 189 \\
\hline
\end{tabular}

Fuente: Elaboración propia

\subsection{Rango de edad cuando ingresaron a los postgrados}

Mayoritariamente $(36,5 \%)$ las personas encuestadas ingresaron a los programas con menos de 30 años. En tanto, quienes se matricularon con edades que oscilan entre 31 y 35 años, 36 y 40 y más de 40 años prácticamente se mantiene en porcentajes similares en torno al 20\%. Al relevar la relación existente entre la edad al matricularse y la elaboración de tesis, se observa un comportamiento errático, donde los estudiantes de menor edad al inscribirse tuvieron una proporción de 0,56 de elaboración de tesis, que disminuye en el segundo rango de edad que va de 31 a 35 años con una proporción de 0,40, para volver a crecer, la proporción de estudiantes con tesis, a medida que aumenta el rango etario (tabla 3).

Al realizar la prueba Chi cuadrado entre la edad al matricularse y la elaboración de tesis no se encontró asociación entre las variables $X^{2}(3, N=102)=4,080, p=0,253$. Sin embargo, los resultados encontrados por Hirschhorn (2012, p.25) no coinciden con lo hallado en esta investigación, pues la edad al momento de ser admitido aparece como una variable de importancia, es decir la gente más joven en la etapa de inscripción posee mayores chances de graduarse y no pertenecer al grupo TMT.

Tabla 3

Número y porcentaje de estudiantes que defendieron la tesis, según grupo etario, Universidad Nacional de Asunción-Paraguay 2004-2014

\begin{tabular}{ccccccc}
\hline \multirow{2}{*}{ Rango etario } & \multicolumn{2}{c}{ Con Tesis } & \multicolumn{2}{c}{ Sin Tesis } & Total & Porcentaje \\
& Fr & $\%$ & Fr & $\%$ & & con tesis \\
\hline Menor a 30 años & 39 & 38 & 30 & 35 & 69 & 56 \\
Entre 30 y 35 años & 17 & 17 & 25 & 29 & 42 & 40 \\
Entre 36 y 40 años & 22 & 21 & 16 & 18 & 38 & 57 \\
Mayor a 40 años & 24 & 24 & 16 & 18 & 40 & 60 \\
Total & 102 & 100 & 87 & 100 & 189 & 53 \\
\hline
\end{tabular}

Fuente: Elaboración propia 


\subsection{Programas de postgrado, según áreas del conocimiento y realización de tesis}

Considerando los programas cursados por las personas encuestadas, el mayor porcentaje $(69,6 \%)$ de quienes no han realizado la tesis se da con estudiantes de las Ciencias Sociales y Humanidades, seguido por quienes cursaron postgrados relacionados con la Ingeniería y Tecnología $(57,1 \%)$. Esto coincide con lo manifestado por Latona y Browne (citado en Hirschhorn, 2012, p. 25), quienes encontraron que estudiantes de Ciencias Sociales, Educación o disciplinas Humanísticas demoraban más en realizar la tesis.

Wainerman y Tuñón (2013) también encontraron esta tendencia y conjeturan que la mayor eficiencia en las Ciencias Exactas y Naturales por sobre las Ciencias Sociales y Humanas se debe a los modos de formación entre una y otra, donde en la primera se reproduce más el

modelo oficio y de la artesanía, dentro de la comunidad de práctica, mediante reglas explícitas e implícitas existentes; mientras que en la segunda, generalmente, el trabajo ocurre en soledad, con la ayuda esporádica del director o directora de tesis y con escaso o ningún contacto con colegas. (p.170)

Atendiendo el promedio en años que insume el proceso de preparación y defensa de la tesis, desde el ingreso al programa de postgrado, quienes han cursado programas relacionados con las Ciencias Agrícolas han insumido mayor tiempo en la finalización de la tesis con 4,8 años en promedio, seguido de quienes han cursado programas de postgrado relacionado a la Ingeniería y Tecnología con 4,4 años en promedio. En tanto, las personas encuestadas que culminaron la tesis en menor cantidad de tiempo cursaron programas de postgrado relacionados a las Ciencias Naturales con 2,8 años en promedio (tabla 4).

Los valores hallados, en esta investigación, serían menores por ejemplo al encontrado por Hirschhorn (2012), Valarino (2010) quien, en el primer caso menciona, en promedio, la realización del trabajo de tesis en 6,6 años; mientras que la segunda autora encontró que en promedio demoraban 9 años. Los resultados permiten ver una heterogeneidad en el grado de eficiencia entre disciplinas, pero es importante remarcar en este sentido, que los programas de postgrados de las diversas facultades o unidades académicas de la Universidad Nacional de Asunción no se rigen por un mismo reglamento de tesis, por lo cual en parte, no resultan comparables entre sí dado que el grado de burocracia o dificultad para obtener el grado pretendido difiere entre unidades académicas. 
Por otro lado, la elaboración del trabajo de tesis encontrado en la presente investigación se halla en promedio, en el tiempo establecido como máximo en el Reglamento General de Postgrado del Rectorado de la Universidad Nacional de Asunción, donde se estipula que los créditos académicos fenecen luego del doble de duración del programa de postgrado. Atendiendo que las maestrías duran en promedio 24 meses, el tiempo insumido por el estudiantado se podría considerar acorde al plazo estipulado.

Tabla 4

Promedio en años que insume la elaboración de tesis, por área del conocimiento, Universidad Nacional de Asunción-Paraguay 2004-2014

\begin{tabular}{lcccccc}
\hline Áreas & \multicolumn{2}{l}{ Con Tesis } & Sin Tesis & Total & $\begin{array}{c}\text { Años } \\
\text { realización } \\
\text { Tesis } \\
\text { (promedio) }\end{array}$ \\
& & & & & & \\
\hline Ciencias Naturales & 49 & 76,5 & 15 & 23,8 & 64 & 2,8 \\
Ingeniería y Tecnología & 9 & 45 & 11 & 57,1 & 20 & 4,4 \\
Ciencias Médicas & 1 & 100 & 0 & 0 & 1 & 3 \\
$\begin{array}{l}\text { Ciencias Agrícolas } \\
\text { Ciencias Sociales y }\end{array}$ & 16 & 100 & 0 & 0 & 16 & 4,8 \\
Humanidades & 27 & 30,3 & 61 & 69,3 & 88 & 3,7 \\
Total & 102 & & 87 & & 189 & \\
\hline
\end{tabular}

Fuente: Elaboración propia

\subsection{Modalidad de trabajo y realización de tesis}

Al indagar la relación existente entre la modalidad del trabajo (parcial/completo) y la elaboración o no de la tesis, los resultados revelan que, al momento de ingresar y cursar el programa de postgrado, el $38,2 \%$ de quienes terminaron la tesis trabajaban a tiempo total y el $61,7 \%$ a tiempo parcial. Atendiendo los resultados de la prueba estadística $X^{2}(1$, $\mathrm{N}=189)=5,93, \mathrm{p}=0,018$ se encontró asociación entre estas variables. Este resultado coincide con Carlino (2005); Martínez et al., (2000) y Valarino, (2000), quienes señalan la variable dedicación laboral como altamente predictora para hallar el suceso esperado, es decir, elaborar la tesis. Además, la importancia de esta variable radica en que se relaciona con otras, como el tiempo de dedicación a la tesis.

\subsection{Relación de la actividad laboral prioritaria y el postgrado cursado}

Es de suponer que una afinidad entre la actividad laboral realizada y el programa de postgrado cursado facilitaría la realización del trabajo de tesis. Por ello, a la pregunta ¿Su 
actividad laboral se relaciona o relacionaba con el programa de postgrado cursado? las respuestas obtenidas dan cuenta de que un 60,7\% de las personas encuestadas que terminaron la tesis manifestaron que su actividad laboral prioritaria se relacionaba con el programa de postgrado cursado, mientras que el $59,7 \%$ de quienes no finalizaron la tesis también manifestaron que la actividad laboral prioritaria y el programa de postgrado estaban relacionados (tabla 5).

Atendiendo la hipótesis inicial planteada donde se espera que si la actividad laboral prioritaria del estudiantado y el programa de postgrado guardan cierta relación favorecería la elaboración de la tesis, no se encontró asociación entre ambas variables $X^{2}(2, N=189)=0,50$, $p=0,776$, con lo cual se puede entender que no hay diferencia entre el estudiante de postgrado que se desempeña laboralmente en un área afín al programa de postgrado y aquel que no lo hace y finalizar la tesis.

Desglosando esta misma variable, actividad laboral en Áreas del Conocimiento y analizando por separado entre quienes tienen la tesis y quienes no la tienen, para el primer caso (con tesis), el mayor porcentaje de personas encuestadas cuya actividad laboral se relaciona con el programa cursado pertenece a las Ciencias Agrícolas (86,7\%), seguido por el Área de Ingeniería y Tecnología. Entre las personas graduadas de las Ciencias Sociales y Humanidades, prácticamente la mitad $(48,1 \%)$ no se desempeña laboralmente en el área del postgrado cursado; no obstante, realizaron la tesis.

Tabla 5

Afinidad entre actividad laboral prioritaria, el programa de postgrado y finalizar la tesis, Universidad Nacional de Asunción-Paraguay 2004-2014

\begin{tabular}{cccc}
\hline Relación actividad laboral/ postgrado & $\begin{array}{c}\text { Con Tesis } \\
(\mathrm{fr})\end{array}$ & $\begin{array}{c}\text { Sin Tesis } \\
(\mathrm{fr})\end{array}$ & $\begin{array}{c}\text { Total } \\
(\mathrm{fr})\end{array}$ \\
\hline Sí & 62 & 52 & 114 \\
No & 12 & 8 & 20 \\
Parcialmente & 28 & 27 & 55 \\
Total & 102 & 87 & 189 \\
\hline
\end{tabular}

Fuente: Elaboración propia

\subsection{Ayudas monetarias o becas}

Consultados si accedieron a ayudas monetarias o becas para solventar el costo del programa de postgrado, el 58,5\% manifestó que No recibió y el 41,5\% que Sí recibió. De quienes manifestaron haber recibido beca, el 54,4\% recibió beca parcial y 45,6 beca total.

Aquí se puede mencionar lo expresado por Luchilo (2010) que "para algunos postgrados especialmente los de orientación más académica, el aporte público a través de 
becas, apoyo a proyectos de investigación y de infraestructura constituye una fuente de recursos indispensable. Para otros, como los posgrados de orientación profesional como las de las áreas de economía, administración, negocios o ciencias de la salud, el pago del estudiantado representa la base de sus ingresos". (p.20). Jaramillo (2009) expresa

el hecho que exista un gran porcentaje de personas que costean su estudios o programas de postgrado se debe a que tanto la política de ciencia, tecnología e innovación así como la de formación de recursos humanos de alto nivel no ha logrado aún una estabilización de crecimiento continuo en la asignación de sus recursos, dependiendo más del ciclo económico y de las coyunturas particulares. (p.134)

Por otro lado, de acuerdo a los resultados de la prueba de hipótesis entre quienes recibieron beca y la probabilidad de finalizar la tesis, se puede concluir con una probabilidad de error de prácticamente cero por ciento que existe asociación entre finalizar la tesis y haber recibido becas o ayudas monetarias $\mathrm{X}^{2}(1, \mathrm{~N}=189)=12,45, \mathrm{p}=0,0001$. Estos resultados coinciden con lo encontrado por Hirschhorn, (2012), quien dice que el estudiantado que accede a la beca tiene $53 \%$ más chances de defender la tesis.

Por otra parte, al analizar la relación existente entre ser varón y recibir beca o ser mujer y haber recibido beca y la probabilidad de realizar la tesis, no se encontró asociación, por lo cual no existen diferencias entre ser varón o mujer, recibir beca y finalizar la tesis $X^{2}(1$, $\mathrm{N}=189$ )=0,0001, $\mathrm{p}=0,989$ ). Sin embargo, Hirschhorn, (2012), encontró asociación significativa entre ser mujer, recibir beca y defender la tesis es decir, donde las mujeres con beca tienen más chances de defender la tesis que los varones con beca.

\subsection{Realizar docencia e investigación}

Un 51,3\% manifestó que no realiza ni docencia ni investigación, en tanto un 35,4\% manifestó que sí hacia docencia universitaria pero no investigación, y tan solo un 6,9\% hacía ambas actividades. El menor porcentaje (3,2\%) de las personas encuestadas manifestó que formaba parte de grupos de investigación y también un 3,2\% que solo hacía investigación en universidades sin hacer docencia. La asociación entre hacer docencia e investigación y la realización de la tesis se mostró altamente significativa $X^{2}(4, N=189)=20,44, p=0,0001$ con lo cual se podría esperar que el hecho de realizar estas actividades contribuye o facilita la elaboración de la tesis. En esta línea, Hirschhorn (2012) encontró que los programas que tienen mayor vínculo institucional, traducido en una mayor vida académica donde la docencia 
y la investigación juegan un rol preponderante del personal docente, presentan mayor eficiencia terminal.

\subsection{Frecuencia de encuentros con el tutor o la tutora de tesis}

Del total de 78 encuestados que han respondido a la pregunta de con qué frecuencia se producían los encuentros con el tutor o la tutora de tesis, un 53,4\% de quienes finalizaron la tesis manifestaron que dichos encuentros fueron de manera mensual, uno por mes; mientras que el $20,5 \%$ manifestó que los encuentros fueron aproximadamente uno cada tres meses y $13,6 \%$ tuvo encuentros prácticamente uno cada seis meses. El resto mantuvo encuentros más esporádicos uno por año o menos con el tutor o la tutora.

Al analizar la asociación entre esta variable y la finalización de la tesis, la misma se mostró significativa, como es de esperar, $\mathrm{X}^{2}(3, \mathrm{~N}=189)=84,6, \mathrm{p}=0,001$, con lo cual se puede entender que una mayor frecuencia de encuentros resulta beneficiosa en el proceso de elaboración de la tesis. Estos resultados coinciden con Hirschhorn (2012), quien encontró que la frecuencia típica de encuentro, en torno a una reunión mensual, resultó altamente significativa en la finalización de la tesis.

\subsection{Líneas de investigación y pertinencia de las líneas}

Se consultó si el programa de postgrado ofreció líneas de investigación, y el 64\% manifestó que Sí y el 36\% que No. Respecto a la pertinencia de las líneas de investigación, mayoritariamente $(56 \%)$ han considerado que fueron pertinentes, en tanto un menor porcentaje de encuestados, 4\%, consideró que estas líneas fueron poco pertinentes. Analizando las variables líneas de investigación y la elaboración de tesis, no se encontró asociación entre ambas $\mathrm{X}^{2}(1, \mathrm{~N}=189)=2,0, \mathrm{p}=0,153$ por lo cual se puede esperar que la explicitación o facilitación de las líneas de investigación no necesariamente ayuda a la finalización de la tesis. Y este resultado es comprensible atendiendo el tipo de programa predominante que cursa el estudiantado de postgrado que responde al tipo profesionalizante, donde la realización de investigaciones es prácticamente nula, siendo la tesis en la mayoría de los casos la única investigación realizada por el estudiante, con lo cual las líneas de investigación no cobran relevancia. En este sentido, Romay (1994) en su estudio El papel de la investigación en los programas de posgrado en educación en México. Estudio de caso de seis programas (1980-1992), confirmó que las tesis continúan siendo el mayor proyecto de investigación que realizan los estudiantes de postgrado, sin embargo, menciona que "la clara 
definición institucional de líneas de investigación parece ser un medio importante para delimitar los campos de investigación en que los estudiantes incursionan" (pp. 180-181).

\subsection{Comité de tesis}

Analizando la elaboración de tesis y la existencia de comité, un 65,3\% de quienes dijeron haber tenido comité elaboraron la tesis (64/101); mientras que entre quienes no hicieron la tesis, el 50\% (44/88) también manifestó que tenía un comité de tesis. No se encontró asociación significativa entre disponer de un comité y la elaboración de tesis $\mathrm{X}^{2}$ (1, $\mathrm{N}=189)=2,84, \mathrm{p}=0,09$. Esto en parte puede explicarse, tomando lo dicho por Wainerman $\mathrm{y}$ Tuñón (2013), respecto a la gran heterogeneidad de los programas de postgrado existentes entre los que predominan los de tipo semiestructurado, caracterizado por la cursada en aulas de módulos o materias, siendo los demás aspectos, que hacen a un programa estructurado como la existencia de un comité de seguimiento de tesis, una consejería de estudios, la realización de cursos complementarios para la elaboración de la tesis entre otros aspectos, prácticamente tratados de soslayo. Por otro lado, la modalidad de los programas de postgrados de tipo personalizado, donde es posible tener una "trazabilidad" del alumnado es la modalidad con menor implementación, dándose quizás de alguna manera y en forma reciente, con aquellos programas de postgrado que han sido fortalecidos por el Consejo Nacional de Ciencia y Tecnología (CONACyT), por lo cual probablemente se debe replantear tanto la existencia como el rol que deben desempeñar estas instancias. La conformación de los Comités o Comisión Asesora de tesis como modelo de gestión de la investigación más participativa, constituye práctica reciente en la UNA, y está en proceso de cristalización, por lo cual los resultados aún no son percibidos por el estudiantado de postgrado.

\subsection{Cursos o talleres de tesis, proyectos de investigación}

Se consideró que la realización de cursos, talleres o entrenamientos en la elaboración de tesis podría aumentar la probabilidad de finalización de la tesis. Las respuestas obtenidas dan cuenta de que un 55\% dijo No haber realizado este tipo de actividad académica, mientras que el restante $44,9 \%$ Sí lo realizó. Del total de encuestados que respondieron haber realizado este tipo de capacitación, un 47,0\% finalizó la tesis; en tanto entre quienes no realizaron este tipo de capacitación, el 59,6\%finalizó la tesis. Estos resultados no permiten, al contrario de lo esperado, hallar una asociación entre estas variables, es decir, realizar la tesis y los cursos o talleres previos $X^{2}(1, N=189)=2,96, p=0,085$. 
Ante esto se puede traer a colación lo dicho por Soto y Salas (2014, p. 2) quienes mencionan que existe una visión burocrática de la investigación que incide negativamente en los procesos de enseñanza de los cursos de metodología, por lo cual se deben desarrollar talleres de investigación pero como experiencia vital académica para el desarrollo de la pasión intelectual y no solo la investigación como medio para alcanzar un producto de graduación.

La experiencia en tutorías como en capacitaciones realizadas en talleres de tesis, permite constatar que el estudiantado acude a estos espacios con conocimientos muy básicos o incluso sin previo conocimiento en esta materia, por lo tanto, el aprovechamiento es muy bajo lo cual se refleja en la dificultad en redactar y finalizar la tesis. Así también, los estudiantes realizan estos cursos sin haber pensado, en muchos casos, aún en el tema de investigación, por lo tanto, no existe un vínculo entre la teoría y la práctica. Así también se puede mencionar el nivel incipiente de calidad metodológica exigido, en algunos casos, para la elaboración de las tesis, por lo cual la persona que está en proceso de elaboración siente baja presión para la excelencia.

Muy similar a la pregunta anterior, se relevó si el encuestado realizó antes o durante la cursada del programa de postgrado cursos de estadística o análisis cualitativo de datos, pues se consideró que podrían facilitar el proceso de elaboración de la tesis. El 60,8\% manifestó que No y 39,1\% que Sí. De quienes manifestaron haber realizado estos tipos de cursos, prácticamente en igual proporción, 38/36, realizó la tesis y no lo hizo. Al analizar la asociación entre la variable realización de cursos de estadística o análisis cualitativo de datos, y elaboración de tesis, nuevamente no se encontró asociación $X^{2}(1, N=189)=3,33$, $\mathrm{p}=0,56$.

Siguiendo en la misma línea de la pregunta anterior, se relevó si mientras cursaba el programa de postgrado, la persona encuestada participó en proyectos de investigación. Un $74,6 \%$ dijo que No, mientras un 25,3\% dijo que Sí participó. Tampoco se encontró asociación entre haber participado en proyectos de investigación y la realización de la tesis $\mathrm{X}^{2}$ (1, $\mathrm{N}=189)=0,49, \mathrm{p}=0,482$ y este comportamiento puede encontrar respuesta nuevamente en el modelo profesionalizante de los programas impartidos donde la investigación no se ve privilegiada. Sin embargo, Hirschhorn (2012) encontró asociación significativa entre la práctica de elaborar proyectos de investigación y la finalización de tesis.

Finalmente, se preguntó si antes de elaborar la tesis, realizó publicaciones. Las respuestas dan cuenta de que el $78,8 \%$ No lo hizo, mientras que el 21,1\% Sí publicó antes 
de realizar la tesis. Del mismo modo que la pregunta anterior, no se halló asociación significativa entre las publicaciones y la realización de la tesis $X^{2}(1, N=189)=0,02$, $p=0,883$. Estos resultados se hallan contestes con la respuesta donde solo el $6,9 \%$ hacía docencia e investigación y un 3,2\% de encuestados formaba parte de un grupo de investigación, ámbitos estos donde la publicación de los trabajos resulta imperativa, reforzando que la tesis constituye para un grupo mayoritario de estudiantes de postgrado el primer "trabajo de investigación" importante que realizan.

\subsection{Motivos de no realización de la tesis}

Focalizándose exclusivamente en quienes no realizaron la tesis $(n=88)$, como principal motivo expresado por el $22,7 \%$ (tabla 6 ), se tuvo el aspecto metodológico, que tiene que ver con no saber hacer o tropezar con dificultad al momento de la redacción, tener inconvenientes con la escritura. Este factor ha sido definido como competencias académicas exiguas por el Canadian Journal of Higher Education y se manifiesta recurrente en trabajos como en los de Delgado Reynaga (s.f.); Hirschhorn (2012); y Rietveldt de Arteaga Vera, (2012). Por otro lado, Peñaloza (2003, p. 147) resalta el carácter "panorámico" con que se imparten muchos programas de postgrado que no favorece la investigación, sumado a todo esto se encuentra la baja capacidad de las propias instituciones de educación superior para generar conocimientos (Rojas, 2008).

La poca preparación metodológica también responde a una estrategia errónea de enseñanza de la metodología de la investigación, donde la materia se imparte bajo una modalidad predominantemente teórica, "de receta", pues en muchas ocasiones y como se mencionó, la persona que cursa un postgrado no posee aún el tema de investigación durante la cursada, en consecuencia se dificulta la conexión entre la teoría y la práctica, de allí que el alumnado no sepa cómo hacer la tesis, limitante reconocida por los propios estudiantes, en las entrevistas:

"Yo creo que la parte de la metodología de la investigación es lo que más se tiene que fortalecer en los postgrados, porque bien sabemos que damos los módulos pero la metodología de la investigación tiene una duración de un mes o un poco más, pero no es suficiente o sea que para mí que la metodología de la investigación se debería acompañar desde el comienzo o sea cuando uno empieza ya la maestría elegir su tema, porque de repente vos tenés la idea pero ese módulo de metodología que tuviste y si era básico luego tu conocimiento, de grado, peor todavía entonces no 
sabemos bien, yo siempre digo que mi tesis de maestría es lo que me permitió a mí ver bien los pasos, de la metodología hay muchas cosas básicas que no comprendemos, y si uno sabe desde el comienzo, es como que vos hiciste toda una ropa y tenés que volver a descoser, tener que hacer todo de nuevo eso para mí fue mortal". (Entrevista 4-30.03.2018).

Por otro lado, Valarino (2000) sostiene que resulta fundamental, para lograr el éxito académico, la preparación y la experiencia del profesorado de investigación, quienes deben desarrollar competencias actitudinales, cognitivas como procedimentales.

La segunda causa de no tesis se halla relacionada a problemas económicos, laborales o familiares con un $20,4 \%$ de respondientes, la tercera tiene que ver con la dificultad de carácter personal, donde un 15,9\% consideró como el factor por el cual no se produce el avance en la tesis. Al interior de esta categoría se consideraron subcategorías siendo la falta de perseverancia (desmotivación)(38,2\%) y la falta de orden y sistematicidad de los avances lo cual genera pérdida, extravío de información y datos $(27,3 \%)$,señaladas de manera prioritaria. También la cuestión personal podría estar relacionada a aspectos psicológicos y sociales del estudiantado, coincidiendo con varios autores como Zerpa (citado en Valarino 2000); Rodríguez (2014); el Canadian Journal of Higher Education (1995) entre otros, aspectos no indagados en la presente investigación.

Otros motivos que impiden la realización de la tesis fueron señalados por un $12,5 \%$, de las personas entrevistadas. Al marcar esta categoría se solicitó explicitar ¿cuáles? y entre las aclaraciones más frecuentes se encontraron retrasos de insumos necesarios para realizar los experimentos, no acceso a ayuda profesional adecuada para procesar datos, cambio en el tema de tesis, viajes al exterior, inicio de otro programa de postgrado, principalmente.

Como otro factor principal que impide finalizar la tesis un $12,5 \%$ de personas encuestadas mencionó las trabas impuestas por la institución donde cursó el programa de postgrado. En este sentido, puede considerarse una traba institucional la desprolijidad metodológica entre los diversos actores (docentes de metodología de la investigación, grupo de tutores, cuerpo de evaluadores internos y externos e integrantes de la mesa evaluadora), lo cual crea un sentimiento de impotencia, estrés y frustración en tesistas, que en ocasiones resultan como las verdaderas causas explicativas del abandono de tesis. Así la entrevista, recogió esta situación: 
"tenés tres evaluadores a quienes se envía el borrador; uno te dice así tenés que hacer, el otro te dice así y te cambia otra vez lo que te dijo el otro, entonces como cada uno, no hay así como un equipo consensuado que te pueda dar una respuesta... allí lo ideal es si tenés 3 evaluadores que se reúnan y se pongan más o menos de acuerdo sobre los puntos para responderle al estudiante porque al recibir yo la respuesta de un evaluador uno me dice tu objetivo está atravesado, el otro me dice tu objetivo está bien, y realmente eso es lo que da la sensación de estrés, porque llega un momento en que el estudiante dice vayan todos a la m....porque ni si entre los evaluadores se ponen de acuerdo por supuesto que hay criterios, indicadores pero que cada uno interpreta a su manera, y yo por ejemplo llegué a un momento en que no sabía a quién hacerle caso, y después lo peor otra vez en tu mesa se sienta totalmente otra gente, que ni siquiera vieron tu trabajo, que ya llegaste allí a la defensa después de muchos filtros e imagínate que otra vez te digan tu objetivo está mal...algo que te corrigieron 10 mil veces y 3 años es una locura" (Entrevista 430.03.2018).

Finalmente, ahondando en las causas, se visualiza que el 6,8\% dijo que es la falta de apoyo del director o directora o del tutor o tutora de tesis; mientras que en menor porcentaje manifestaron que no saben sobre qué tema investigar y que no acceden a tutoría con 5,7\% y $3,4 \%$ respectivamente. Algunos factores aquí mencionados coinciden con los resultados hallados por Ramos y Sotomayor (2008), quienes como razones principales para no hacer la tesis encontraron: los trámites engorrosos de la institución $(60,6 \%)$, falta de recursos $(57,8 \%)$ y la dificultad para encontrar problemas de investigación (8,5\%).

\section{Tabla 6}

Principales motivos por los cuales los estudiantesno finalizan la tesis, Universidad Nacional de Asunción-Paraguay 2004-2014

\begin{tabular}{lcc}
\hline Motivos & Frecuencia & Porcentaje \\
\hline No sabe cómo hacer, le cuesta escribir (aspecto metodológico) & 20 & 22,7 \\
Enfrentó problemas económicos, laborales o familiares & 18 & 20,4 \\
Dificultad personal & 15 & 15,9 \\
Otros & 11 & 12,5 \\
La institución le pone trabas & 11 & 12,5 \\
Falta de apoyo del director o directora o tutores de tesis & 6 & 6,8 \\
No sabe sobre qué tema investigar & 5 & 5,7 \\
No accede a tutores de tesis & 3 & 3,4 \\
Total & 87 & 100 \\
\hline
\end{tabular}

Fuente: Elaboración propia 


\section{Conclusiones}

La investigación permitió obtener respuestas a la interrogante planteada respecto a cuáles son los factores que impiden la realización de la tesis en el alumnado de programas de postgrado de la Universidad Nacional de Asunción. En este sentido, los hallazgos dan cuenta de 4 factores que estarían asociados a este fenómeno, que son: la modalidad de trabajo (tiempo total/tiempo parcial), haber recibido o no ayudas monetarias o becas, realizar docencia e investigación en universidades y la frecuencia de encuentro con el tutor o la tutora. En tanto, quienes no terminaron la tesis manifestaron como principal motivo los problemas metodológicos (no saben cómo hacer), seguido de los problemas económicos, laborales o familiares a más de los problemas de carácter personal y aspectos como la traba institucional, se mencionó también por este grupo la falta de apoyo por parte del cuerpo de tutores o directamente el no acceso a uno.

No se encontró asociación entre finalizar o no la tesis y las siguientes variables: sexo, edad al ingreso en el postgrado, relación de la actividad laboral prioritaria y el programa de postgrado cursado, líneas de investigación, comité o asesores de tesis, cursos o talleres de tesis o cursos de estadística o análisis cualitativo de datos, la participación en proyectos de investigación y el haber realizado publicaciones.

Quedó relevada, sin embargo, la condición multifactorial y entrelazada del Síndrome TMT, por lo cual se debe realizar una adecuación tanto institucional-académica como personal del estudiantado (transición de estudiante a tesista), al momento de ofertar programas de postgrado e ingresar a ellos. Un acompañamiento cercano tanto de tutores y tutoras como de una comunidad de práctica se considera útil para propender a la eficiencia terminal, así como la dotación de condiciones externas como una fuente de financiamiento asegurada.

Futuros trabajos en esta línea deberían ahondar la temática en relación con el cuerpo de tutores y el relacionamiento y mecanismo de acción de estos, en el marco de los programas de postgrado y al interior de las unidades académicas, dado el rol preponderante que juegan en la finalización de la tesis. Asimismo y considerando la dificultad que se tuvo en la presente investigación en el acceso a datos que muestren de manera fehaciente, estadísticas sobre el alumnado de postgrado, las unidades académicas deben iniciar un proceso ordenado y continuo de actualización de la información que les permita verificar el estado real de quienes ingresan y egresan de los programas y las dificultades con las cuales tropiezan en la elaboración del trabajo de tesis. 
Por otra parte, es oportuno instalar el debate respecto a cómo se imparten las cátedras de metodología de la investigación en postgrados y cómo aprende el alumnado a hacer ciencia, especialmente en las maestrías y doctorados de tipo académico, donde la exigencia de creación de saberes constituye la razón de estos programas, y donde las clases tipo receta "sin ingredientes" parece haber sido la modalidad imperante, lo cual no permite que el alumnado adquiera habilidades ni cognitivas ni prácticas.

\section{Referencias}

Carlino, Paula. (2005). La experiencia de escribir una tesis: contextos que la vuelven más difícil. Anales del Instituto de Lingüística, 14(1), 41-62.

Díaz de Rada, Vidal. (2012). Ventajas e inconvenientes de la encuesta por Internet. Papers, 97(1), 193-223.

Hirschhorn, Alicia Nora. (2012). Factores que facilitan y dificultan la culminación de la Tesis. Análisis comparado de tres Escuelas de Postgrado en Ciencias Agropecuarias (Argentina) (Tesis de Maestría, inédita). Universidad Nacional de Mar del Plata, Mar del Plata, Argentina.

Jaramillo, Hernán. (2009). La formación de posgrado en Colombia: maestrías y doctorados. Ciencia, Tecnología y Sociedad, 13(5), 131-155.

Locke, Leslie Ann y Boyle, Melanie. (2016). Avoiding the A.B.D. Abyss: A Grounded Theory Study of a Dissertation-Focused Course for Doctoral Students in an Educational Leadership Program. The Qualitative Report, 21(9), 1574-1593.

Luchilo, Lucas (Ed.). (2010). Formación de posgrado en América Latina: políticas de apoyo, resultados e impactos. Buenos Aires: Eudeba.

Martínez-González, Adrián; Urrutia-Aguilar María Ester; Martínez Franco Isabel; Ponce Rosas Raul y Gil de Miguel, Ángel. (2003). Perfil del estudiante de posgrado con éxito académico de la UNAM. Tarbiya, 32(1), 133-145.

Narváez Serra Jellicy, Burgos Tovar, José. (2011).La productividad investigativa del docente universitario. Orbis. Revista Científica Ciencias Humanas, 6(18), 116-140.

Odena, Oscar y Burgess, Hilary. (2017). How doctoral students and graduates describe facilitating experiences and strategies for their thesis writing learning process: a qualitative approach. Studies in Higher Education, 42(3), 572-590.

Peñaloza Ramella, Walter. (2003). Políticas y Enfoques del Currículo y la Didáctica para el posgrado en el Perú. En O. A. García (Comp), Hacia una nueva Universidad en el Perú, (pp. 145-154). Lima: Fondo Editorial- UNESCO-IESALC. 
Pereira, Zulay. (2011). Los diseños de método mixto en la investigación en educación: Una experiencia concreta. Revista Electrónica Educare, 15(1), 15-29.

Ramos Rodríguez, Mariana. y Sotomayor, Renzo. (2008). Realizar o no la tesis: razones de estudiantes de medicina de una universidad pública y factores asociados. Rev. Perú Med Exp Salud Pública, 25(3), 322-324.

Rietveldt De Arteaga, Francis y Vera Guadrón Luis. (2012). Factores que influyen en el proceso de elaboración de la tesis de grado. Omnia, 18(2), 109-122.

Rodríguez, Addy. (2014). Factores que dificultan titularse de una universidad mexicana. Cuadernos de Investigación Educativa, 5(20), 117-127.

Rojas Betancur, Héctor Mauricio. (2008).El problema regional de la formación de jóvenes investigadores en el nivel de pregrado en las instituciones de educación superior del departamento del Tolima. (Tesis de Doctorado, inédita).Universidad de Manizales, Manizales, CO.

Romay, María de la Luz. (1994). El papel de la investigación en los programas de posgrado en educación en México. Estudio de caso de seis programas (1980-1992).Revista Latinoamericana de Estudios Educativos, 24(3y4), 171-188.

Soto Acosta, Willy y Salas Ocampo, Luis Diego. (2014). Entre la Tesis y la Investigación en Ciencias Sociales: "Apuntes de Didáctica en un contexto de Cambio". Ponencia presentada al IV Encuentro Latinoamericano de Metodología de las Ciencias Sociales, Heredia, Costa Rica. Recuperado de: http://www.memoria.fahce.unlp.edu.ar/trab eventos/ev.8230/ev.8230.pdf

Soto, Carmiña; Velázquez, Graciela y Samudio Margarita. (2014). Percepción del impacto de los cursos de doctorados y maestrías en el desarrollo profesional de los graduados de la Universidad Nacional de Asunción. Estudio Piloto. Memorias del Instituto de Investigaciones en Ciencias de la Salud, 12(2), 68-81.

Valarino, Elizabeth. (2000). Tesis a tiempo. Barcelona: Carnero.

Wainerman, Catalina y Tuñón, Candela. (2013). La eficiencia de los programas doctorales y su evaluación. Revista Argentina de Educación Superior, 5(6), 167-188. 
Revista indizada en
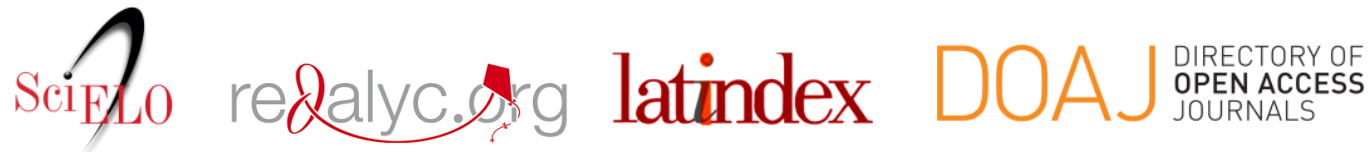

Distribuida en las bases de datos:

๑ Dialnet

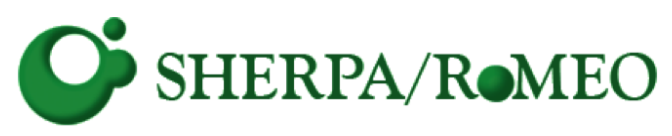

REDIB

Red Iberoamericana

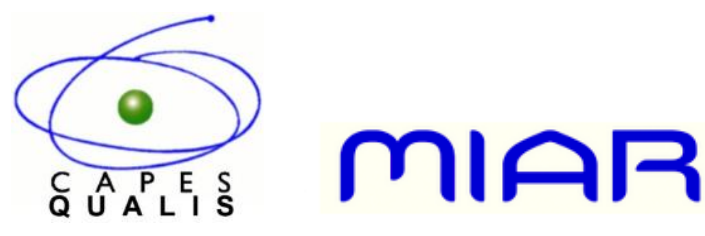

